\title{
Effectiveness of dabigatran etexilate for thromboprophylaxis of mechanical heart valves
}

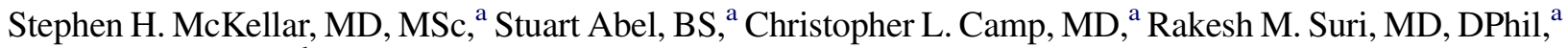 \\ Mark H. Ereth, MD, ${ }^{b}$ and Hartzell V. Schaff, MD $^{\mathrm{a}}$
}

\begin{abstract}
Objective: Warfarin reduces risk of stroke in patients with mechanical heart valves but increases risk of hemorrhage and is difficult to use. Dabigatran etexilate, a new oral direct thrombin inhibitor, is safe and effective in reducing risk of stroke among patients with atrial fibrillation. No data exist in the setting of mechanical heart valves. We tested the hypothesis that dabigatran etexilate is as effective as heparin for thromboprophylaxis of mechanical valves in a porcine heterotopic aortic valve model.
\end{abstract}

\begin{abstract}
Methods: Thirty swine underwent implantation of modified bileaflet mechanical valved conduit bypassing the ligated, native descending thoracic aorta. Animals randomly received no anticoagulation $(n=10)$, enoxaparin $2 \mathrm{mg} / \mathrm{kg}$ subcutaneously twice daily $(\mathrm{n}=10)$, or dabigatran etexilate $20 \mathrm{mg} / \mathrm{kg}$ orally twice daily. Primary end point was amount of valve thrombus at 30 days. Secondary end points included quantitative measurement of platelet deposition on valve prosthesis, thromboelastography, and hemorrhagic and embolic events.
\end{abstract}

Results: At 30 days, we observed $638 \pm 895 \mathrm{mg}$ thrombus in no anticoagulation group, $121 \pm 128 \mathrm{mg}$ in enoxaparin group, and $19 \pm 31 \mathrm{mg}$ in dabigatran etexilate group $(P=.01$ enoxaparin vs dabigatran etexilate). Fewer platelets were deposited on valves in dabigatran etexilate group $\left(2.7 \times 10^{8}\right)$ than in enoxaparin group $\left(1.8 \times 10^{9}\right.$, $P=.03)$. No major or occult hemorrhagic or embolic events were observed. By thromboelastographic analysis, dabigatran etexilate produced less prolongation of $\mathrm{K}$ value $(P=.01)$ and less decreases in angle $(P=.01)$ and maximum amplitude $(P=.001)$ than enoxaparin.

Conclusions: Dabigatran etexilate is as effective as enoxaparin for short-term thromboprophylaxis of mechanical valves. It prevents valve thrombus and platelet deposition at 30 days without increased adverse events. These promising results serve as a foundation for prospective clinical trials with dabigatran etexilate as an alternative to warfarin in patients with bileaflet mechanical aortic valves. (J Thorac Cardiovasc Surg 2011;141:1410-6)

There is increasing interest in using new anticoagulation strategies for patients with mechanical heart valves because of the hemorrhagic and thromboembolic complications associated with long-term anticoagulation with warfarin. ${ }^{1}$ Several new classes of medications have been developed for long-term anticoagulation ${ }^{2,3}$ and have been studied clinically for conditions such as atrial fibrillation ${ }^{4,5}$ and prevention and treatment of deep venous thrombosis after orthopedic surgery. ${ }^{6,7}$

Dabigatran etexilate is a novel, orally administered direct thrombin inhibitor that has proved safe and effective for treating deep venous thrombosis ${ }^{8}$ and preventing stroke in patients with chronic atrial fibrillation. ${ }^{5}$ It has not, however,

From the Divisions of Cardiovascular Surgery ${ }^{\mathrm{a}}$ and Cardiovascular Anesthesia, Mayo Clinic, Rochester, Minn.

Disclosures: Authors have nothing to disclose with regard to commercial support.

Read at the C. Walton Lillehei Forum of the 90th Annual Meeting of The American Association Thoracic Surgery, Toronto, Ontario, Canada, May 2-5, 2010.

Received for publication April 29, 2010; revisions received Sept 9, 2010; accepted for publication Feb 9, 2011; available ahead of print March 23, 2011.

Address for reprints: Hartzell V. Schaff, MD, Mayo Clinic, 200 First St, SW, Rochester, MN (E-mail: schaff@mayo.edu).

0022-5223/\$36.00

Copyright (c) 2011 by The American Association for Thoracic Surgery doi:10.1016/j.jtcvs.2011.02.011 been tested in the setting of thromboprophylaxis of mechanical valve prostheses. Unlike warfarin, dabigatran etexilate has a rapid clinical onset with a predictable dose response. Additionally, there are no known food or drug interactions, and it does not require frequent monitoring for therapeutic effect. Its half-life is approximately 12 hours, and other than the active form (dabigatran), the drug does not have active metabolites and is eliminated through renal and intestinal routes.

We hypothesized that dabigatran etexilate would provide equal thromboprophylaxis of mechanical heart valves as standard anticoagulation strategies. We tested this hypothesis in porcine model of heterotopically placed mechanical aortic valves.

\section{MATERIALS AND METHODS \\ Animal Model}

Approval for this study was obtained from the animal care and use committee of the Mayo Foundation. Adult swine underwent heterotopic mechanical aortic valve placement as previously reported. ${ }^{9}$ The model uses a modified bileaflet aortic valved conduit (St Jude Masters Series; St Jude Medical, Inc, Minneapolis, Minn), which bypasses the ligated, native descending thoracic aorta and directs all blood flow distal to the left subclavian artery across the mechanical valve in the newly constructed neoaorta (Figure 1). 


\section{Abbreviation and Acronym \\ APTT $=$ activated partial thromboplastin time}

\section{Study Design}

Dosing study. Dabigatran etexilate is a novel, orally administered prodrug of the direct thrombin inhibitor dabigatran. Unlike warfarin, it has a rapid clinical onset with a predictable dose response. Additionally, there are no known food or drug interactions, and it does not require frequent monitoring for therapeutic effect. Its half-life is approximately 12 hours, and it has no other active metabolites. Dabigatran is predominantly eliminated by renal excretion. ${ }^{10}$

To identify the most effective doses of dabigatran etexilate and enoxaparin in swine, we first performed a dosing study. Animals were dosed with either dabigatran etexilate or enoxaparin, and appropriate hematologic assay samples were drawn at $0,0.5,1,2,4,8,12,24,36,48$, and 72 hours. ${ }^{11}$ Our aim was to find the doses of dabigatran etexilate and enoxaparin that corresponded to therapeutic levels in our strain of animals. For dabigatran etexilate, we aimed to increase the activated partial thromboplastin time (APTT) 2 to 2.5 times normal. For enoxaparin, we sought an anti-Xa level of at least 0.6 at 4 hours. ${ }^{12}$

In vivo thromboprophylaxis of mechanical valves. Specific details regarding this model of heterotopic aortic valve prostheses have been reported elsewhere. ${ }^{9,13}$ Briefly, 30 swine were randomly sorted into 3 treatment arms of postoperative anticoagulation. These treatments consisted of no anticoagulation $(n=10)$, enoxaparin (Sanofi Aventis US, Bridgewater, NJ) at $2.0 \mathrm{mg} / \mathrm{kg}$ administered subcutaneously twice daily $(\mathrm{n}=10)$, and dabigatran etexilate at $20 \mathrm{mg} / \mathrm{kg}$ by mouth twice daily $(\mathrm{n}=10)$. The clinical formulation of dabigatran etexilate (small tartaric acid pellets coated with drug in capsules) was used in the

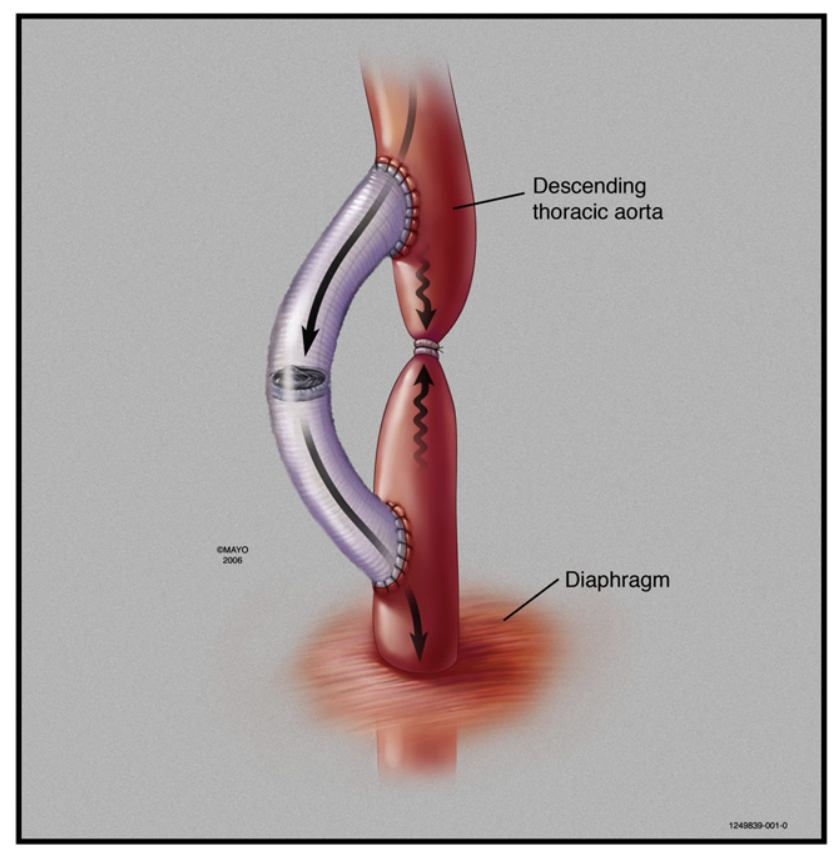

FIGURE 1. Heterotopic aortic valve model demonstrating the modified valved conduit (St Jude Masters Series; St Jude Medical, Inc, Minneapolis, Minn) as it bypasses the ligated native descending thoracic aorta (used with permission from Mayo Foundation for Medical Education and Research). study (Boehringer Ingelheim Pharma GmbH \& Co KG, Biberach Germany). The low-molecular weight heparin enoxaparin was used as the standard for anticoagulation because of the difficulty in maintaining a therapeutic window with warfarin in swine. ${ }^{14}$ Additionally, lowmolecular weight heparins are used to bridge patients to warfarin anticoagulation and as an alternative to warfarin for some patients unable to take warfarin. ${ }^{15,16}$ These doses were determined from the results of our dosing studies. Animals received their assigned treatment medication beginning on postoperative day 1 .

\section{End Points}

Valve thrombus. The primary end point of this study was the amount of valve thrombus at 30 days. ${ }^{16}$ Animals were killed while under general anesthesia after systemic heparinization, at which time valve thrombus was measured and reported as mean $\pm \mathrm{SD}$ in milligrams. Only thrombus on the valve was measured, and this was done by investigators blinded to the treatment arm (S.H.M.).

Platelet deposition. We measured the amount of platelet deposition on the valve prostheses as another quantitative method of assessing thrombus burden and effects of different anticoagulation regimens. Platelet deposition on the mechanical prosthesis in the chronic study was assessed with autologous platelet labeling with indium 111 as previously described. ${ }^{13,17,18}$ After explantation but before quantitation of thrombus burden, the valve was placed into a scintillation radiocounter (Capintec, Inc, Ramsay, NJ). Background radioactivity and the amount of radioactivity from $210-\mathrm{mL}$ tubes of blood was obtained when each animal was killed. By assessing the animal's platelet count and radioactivity obtained from the tubes of blood, the average amount of radioactivity per platelet was determined. This was used to calculate the number of platelets on the prosthesis according to the following equation: Number of platelets on valve prosthesis $=$ Valve radioactivity/Platelet radioactivity

Hemorrhagic and thromboembolic complications. Secondary end points were obvious or occult hemorrhagic and thromboembolic complications. Animals were observed daily for hemorrhagic complications (obvious gastrointestinal hemorrhage) and for thromboembolic complications (neurologic deficits or lower extremity or bowel ischemia). Additionally, kidneys were grossly and microscopically inspected when each animal was killed to search for gross and microembolic events. Occult hemorrhage was sought for as animals underwent serial blood draws to measure serum and fecal hemoglobin concentrations. Occult hemorrhagic events were defined as a drop in serum hemoglobin of at least $2 \mathrm{~m} / \mathrm{dL}$ or an abnormally high fecal hemoglobin level (>2 mg hemoglo$\mathrm{bin} / \mathrm{g}$ of stool).

Coagulation profile. We used several hematologic and anticoagulation assays to assess the effects of dabigatran etexilate on the coagulation system. At baseline (before valve implantation), on days 10 and 20, and when the animal was killed (30 days), we performed a complete blood cell count; measured prothrombin time, APTT, fibrinogen, and anti-Xa levels; and performed thromboelastography. We used the TEG 5000 device with native and kaolin cups and pins (Haemoscope Corporation, Niles, Ill). Measurements obtained included R time (minutes), K time (minutes), angle (degrees), and maximal amplitude ( $\mathrm{mm})$. R time measures time to initial fibrin formation, $\mathrm{K}$ time measures time to strong clot formation and cross-linking, angle measures the speed of clot strengthening, and maximum amplitude measures final clot strength. We included thromboelastography because it evaluates the entire coagulation system and is becoming more widely used in managing cardiovascular surgical patients.

\section{Statistical Analysis}

Valve thrombus and number of platelets deposited on valve prosthesis are reported as mean $\pm \mathrm{SD}$ in milligrams and were treated as continuous variables and compared with the Kruskal-Wallis test for heterogeneity. 


\section{RESULTS}

\section{Dosing Study}

Administration of dabigatran etexilate at $20 \mathrm{mg} / \mathrm{kg}$ orally twice daily reproducibly increased the APTT to 2 to 2.5 times normal (Figure 2, A). Administration of enoxaparin at $2.0 \mathrm{mg} / \mathrm{kg}$ subcutaneously twice daily reproducibly increased anti-Xa levels to at least 0.6 (Figure 2, B). These doses were used for the remainder of the study.

\section{Measurement of Anticoagulation}

Serial hematologic assays confirmed appropriate dose and drug effects for both treatment arms. The APTT increased in animals treated with dabigatran etexilate, and there was also a significant increase in the prothrombin time. (Figure 3, $A$ and $B$ ). As expected, we observed increased anti-Xa levels at all time points for animals receiving enoxaparin relative to the no anticoagulation and dabigatran etexilate groups (Figure 3,C). We observed significantly less circulating fibrinogen in the dabigatran etexilate group at all 3 time points of the study (Figure 3, D).

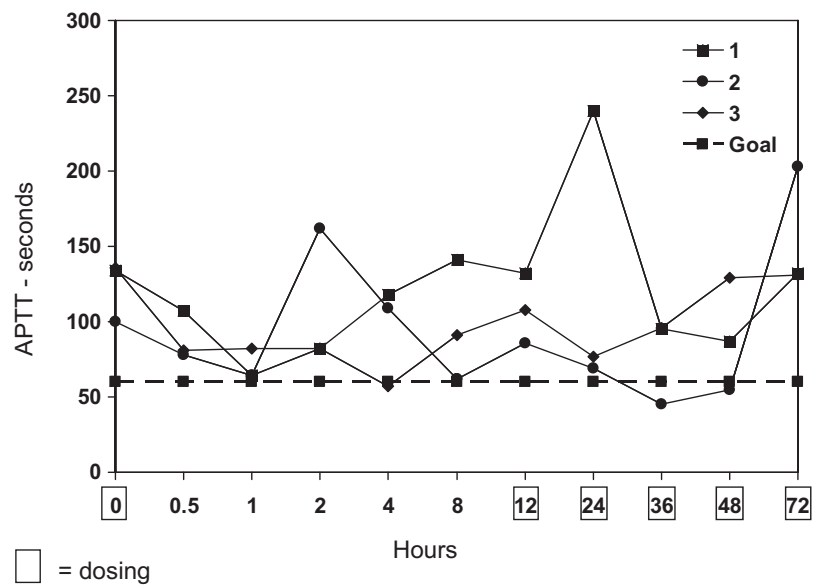

A

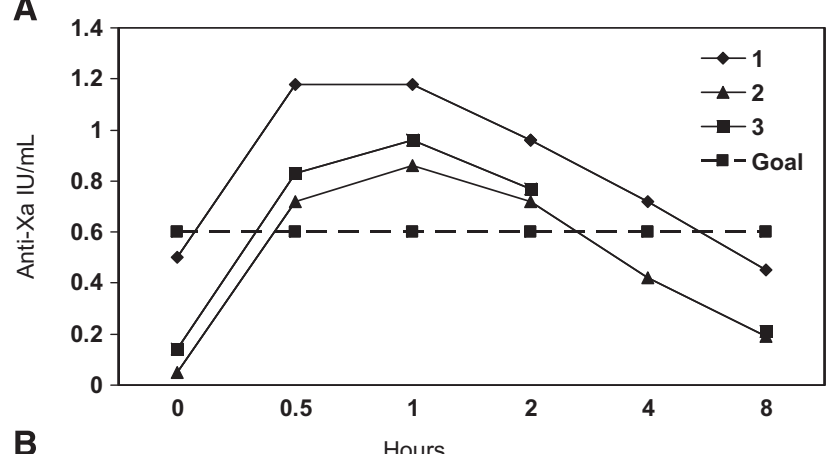

FIGURE 2. Results of dosing study for enoxaparin and dabigatran etexilate for 3 animals. A, For dabigatran etexilate, the goal was an activated partial thromboplastin time (APTT) of 60 seconds (2-2.5 times normal). $\mathrm{B}$, The goal for enoxaparin was an anti-Xa level of 0.6 at 4 hours after dosing.
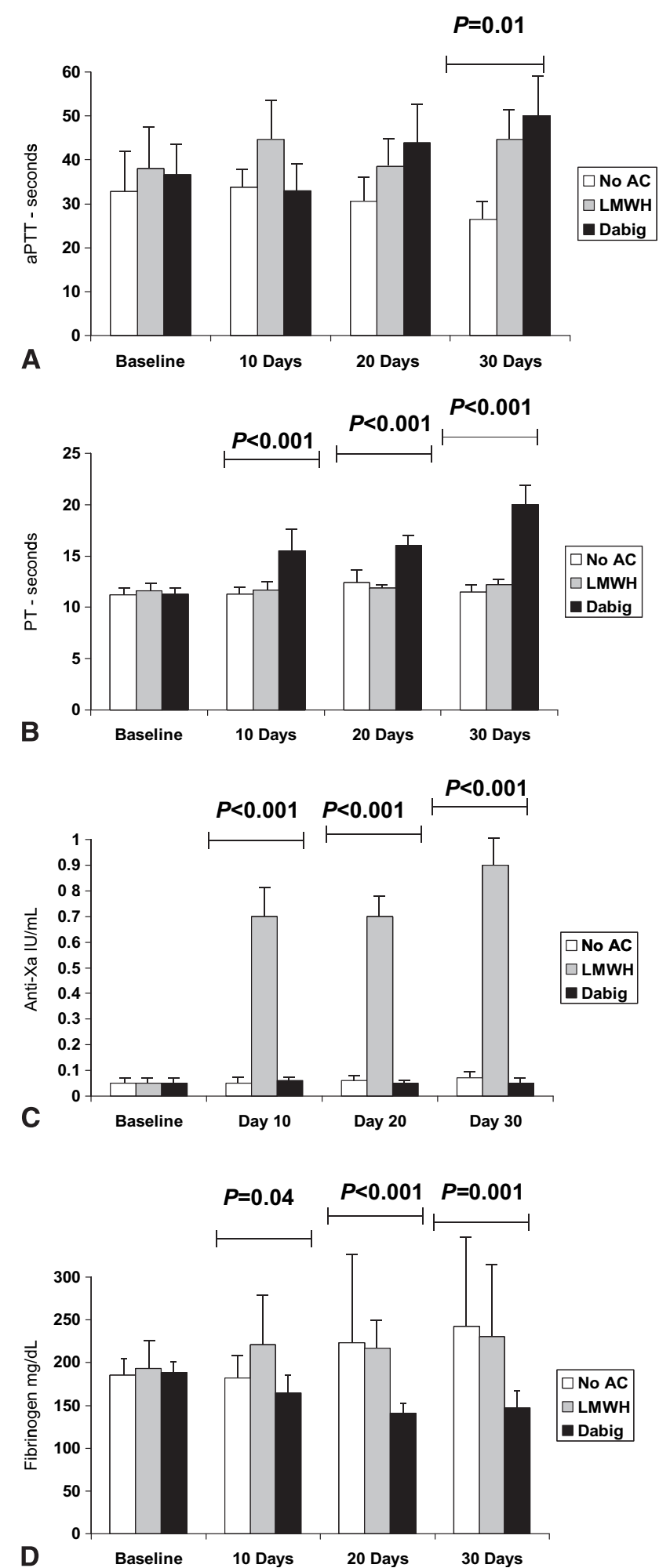

FIGURE 3. Coagulation values throughout study for each group. A, Mean activated partial thromboplastin time $(a P T T)$. B, Mean prothrombin time $(P T)$. C, Mean anti-Xa. D, Mean fibrinogen. No AC, No anticoagulation; $L M W H$, low-molecular weight heparin; Dabig, dabigatran etexilate. 


\section{Valve Thrombus}

There was 1 premature death in the no anticoagulation group as a result of overwhelming sepsis on postoperative day 6. When the animals were killed, mean thrombus weight was statistically different between the groups. We observed $638 \pm 895 \mathrm{mg}$ of thrombus with no anticoagulation, $121 \pm 128 \mathrm{mg}$ with enoxaparin, and $19 \pm 31 \mathrm{mg}$ with dabigatran etexilate $(P=.04$; Figure $4, A)$. This represented a 30 -fold decrease in mean valve thrombus for the dabigatran etexilate group relative to the no anticoagulation group. Comparing the 2 treatment groups, we found significantly less thrombus on valves among animals receiving dabigatran etexilate $(P=.02$; Figure $4, B)$. Similarly, the mean number of platelets deposited on the valve prosthesis was lower in the dabigatran etexilate group $\left(2.7 \times 10^{8}\right)$ than in the enoxaparin group $\left(1.8 \times 10^{9},(P=.03\right.$; Figure $4, C)$.
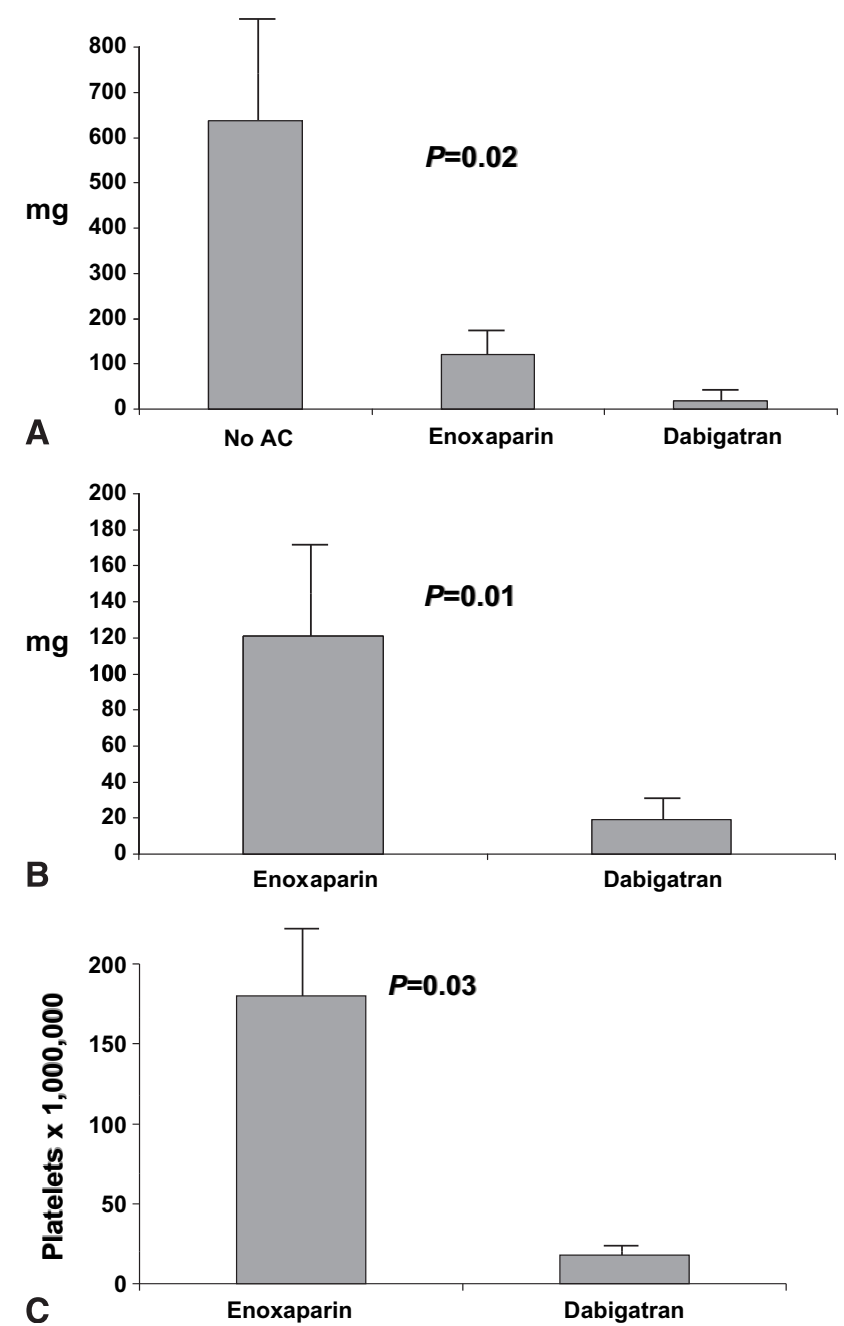

FIGURE 4. A, Mean valve thrombus in milligrams ( \pm SEM) for each group at 30 days. B, Mean valve thrombus for the 2 treatment arms at 30 days $(P=.03)$, demonstrating that the animals that received dabigatran etexilate had the least valve thrombus development. C, Mean platelet deposition on valves for the 2 treatment arms. No $A C$, No anticoagulation.
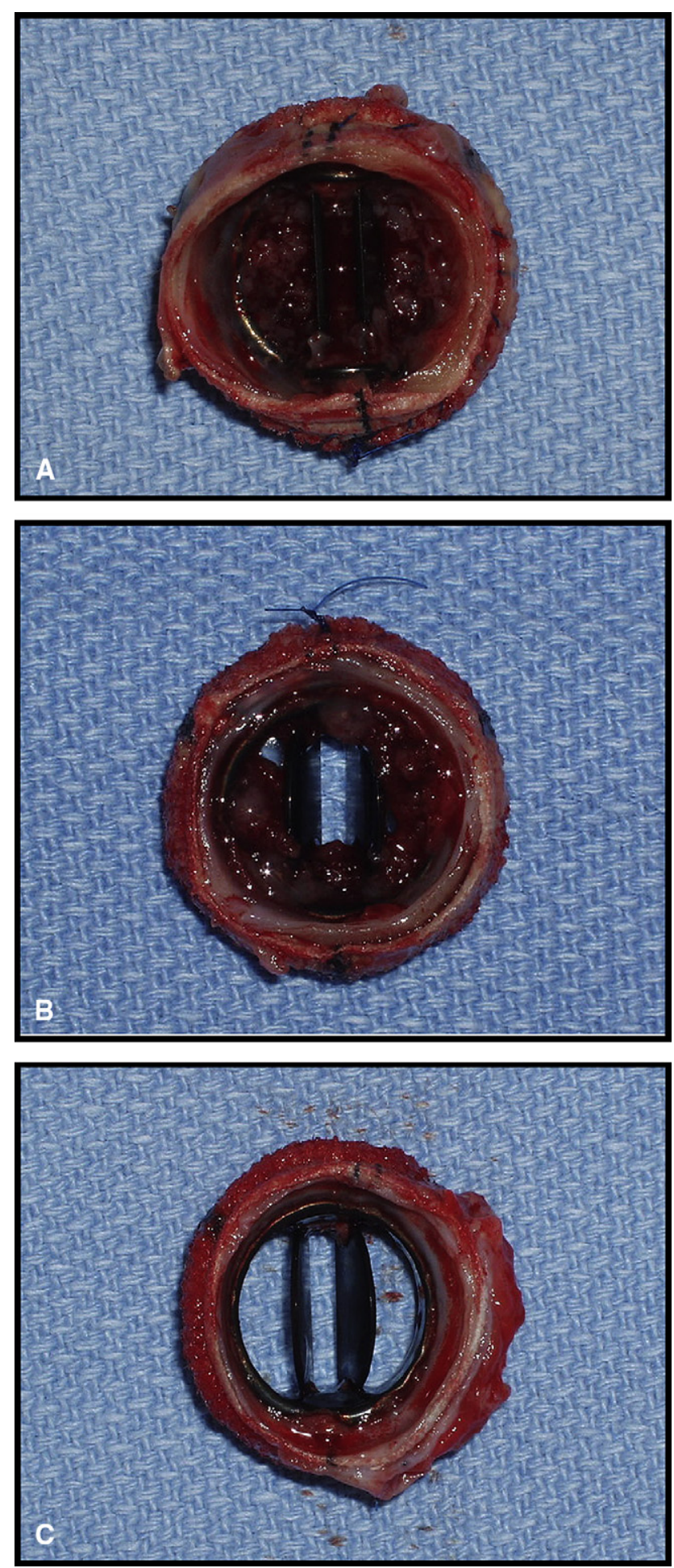

FIGURE 5. Postmortem photographs of explanted valves at 30 days. A, Representative valve from the no anticoagulation group. B, Representative valve from the enoxaparin group. $\mathrm{C}$, Representative valve from the dabigatran etexilate group. 


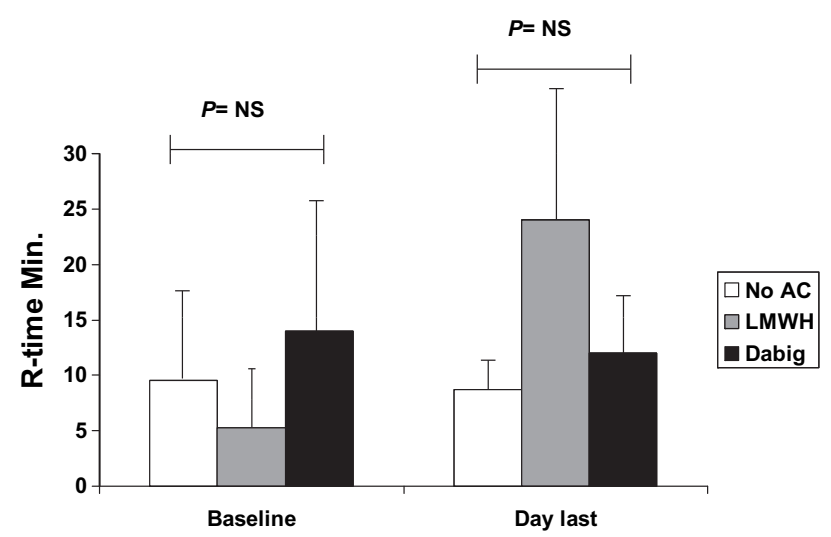

A

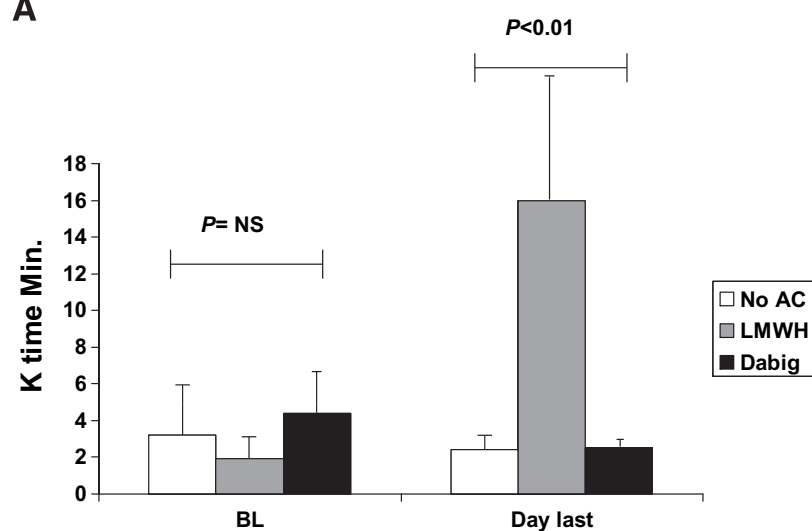

B

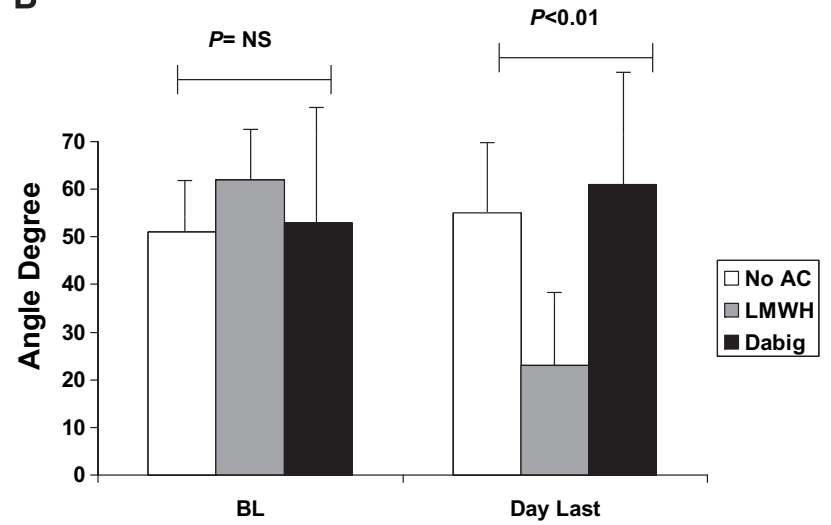

C
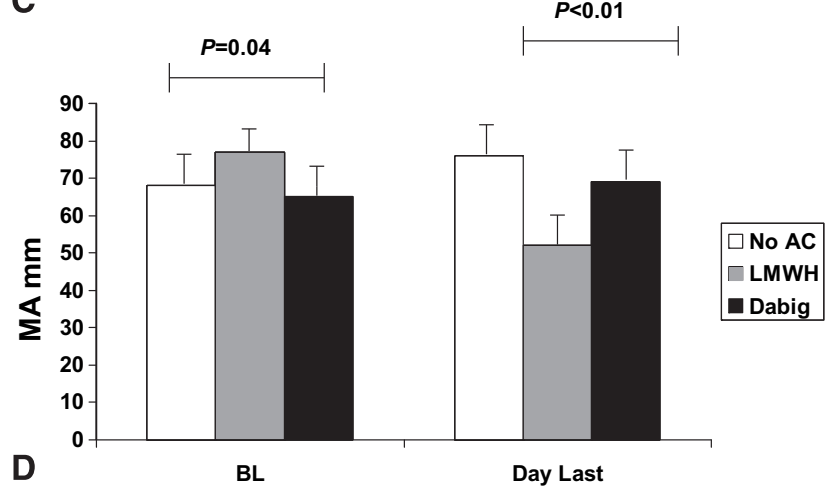

FIGURE 6. Thromboelastographic data for the 3 groups. A, Mean R time (minutes), representing rate of initial fibrin formation. B, Mean $\mathrm{K}$ time
Representative postmortem photographs of explanted valves from the no anticoagulation, enoxaparin, and dabigatran etexilate groups are shown in Figure 5.

\section{Thrombotic and Hemorrhagic Complications}

There were no obvious hemorrhagic or thromboembolic complications. Similarly, there was no evidence of occult bleeding in any of the groups according to serum or fecal hemoglobin assays.

\section{Thromboelastography}

Native and kaolin thromboelastographic assays were performed at baseline and during anticoagulation. We observed that at least in vivo the thromboelastographic coagulation profile ( $\mathrm{R}$ and $\mathrm{K}$ times, angle, and maximum amplitude) in animals receiving dabigatran etexilate looked more like the profiles obtained from animals with no anticoagulation than like those of animals receiving enoxaparin (Figure 6). This was true for both kaolin and native thromboelastographic assays (native data not shown), suggesting adequate anticoagulation despite a normal-appearing thromboelastographic profile.

\section{DISCUSSION}

The principal finding of this study is that the novel direct thrombin inhibitor dabigatran etexilate is effective for thromboprophylaxis of mechanical heart valves in swine. This is the first application of dabigatran etexilate for anticoagulation of mechanical heart valves. In this short-term animal study, treatment with dabigatran etexilate was superior to anticoagulation with heparin and led to the lowest amounts of valve thrombus and platelet deposition at 30 days.

The concept of dabigatran etexilate as a replacement for warfarin for long-term anticoagulation in patients with mechanical valves is promising. Recent clinical trials evaluating dabigatran etexilate for venous thromboembolism and atrial fibrillation showed equivalent or superior efficacy with similar safety profiles. Connolly and colleagues ${ }^{5} \mathrm{dem}-$ onstrated equivalent rates of thromboembolic and hemorrhagic events relative to warfarin for patients with chronic atrial fibrillation. ${ }^{5}$ Similarly, Schulman and associates ${ }^{8}$ reported equivalent safety and efficacy to warfarin in preventing recurrent deep venous thrombosis after orthopedic joint replacement. Similar results were observed for prevention of primary deep venous thrombosis relative to low-molecular weight heparin after orthopedic surgery. ${ }^{19,20}$ If the

(minutes), representing time to strong clot formation and cross-linking. $\mathrm{C}$, Mean angle (degrees), representing speed of clot strengthening. D, Mean maximum amplitude (millimeters), representing final clot strength. $N S$, Not significant; No AC, no anticoagulation; $L M W H$, low-molecular weight heparin; Dabig, dabigatran etexilate. 
pattern of safety and efficacy of dabigatran etexilate can be extended to patients with mechanical valve prostheses, this would represent a major step forward in clinical care.

Unlike warfarin, dabigatran etexilate has rapid in vivo activation and no known drug or food interactions. It has a rapid onset of 2 to 3 hours after ingestion and has a half-life of about 15 hours. It is given twice daily for chronic conditions and does not require invasive blood monitoring as does warfarin. ${ }^{21}$ In addition, the liver toxicity reported for earlier direct thrombin inhibitors has not been observed in these clinical trials. Dabigatran etexilate carries many advantages relative to warfarin: it has no food or drug interactions, does not require routine blood testing, and has a short half-life. It could thus dramatically improve the quality of life in patients who need long-term anticoagulation and could prove safer should emergency surgery be needed. Dabigatran etexilate, like any new drug, requires further studies to determine the best indications for patients with mechanical valves. Whether direct thrombin inhibitors can replace warfarin in selected patients undergoing longterm anticoagulation or whether its use is best reserved for bridging patients to anticoagulation with warfarin needs to be tested in clinical trials.

The thromboelastographic data were unexpected, because animals receiving dabigatran etexilate had the least amount of thrombus and platelet deposition but the thromboelastographic profile more closely resembled that of animals receiving no anticoagulation (Figure 6). Specifically, the times to fibrin and clot formation ( $\mathrm{R}$ and $\mathrm{K}$ times) and the measurements of clot strength (angle and maximal amplitude) were nearly identical to those in control animals. This paradoxic observation suggests ex vivo preservation of the hemostatic axis cascade while providing excellent anticoagulation in vivo. Additionally, we found that prothrombin time had better correlation than APTT to the degree of valve thrombus at 30 days. This is in contrast to results of different assays with human plasma, in which the prothrombin time was quite insensitive to dabigatran etexilate. ${ }^{18}$ To our knowledge, this is the first time that dabigatran etexilate has been evaluated in swine and evaluated with thromboelastography. Our unexpected observations warrant further investigation and may reflect species variation or assay sensitivity to a new class of anticoagulant agents. We were also intrigued by the decreased levels of circulating fibrinogen in dabigatran etexilate-treated animals at all points during the study (Figure 3,D). This may have contributed to less valve thrombus formation because less fibrinogen was available for fibrin formation. This finding also requires further study.

The primary limitation of this study lies in the comparison between dabigatran etexilate and enoxaparin rather than warfarin, which is used clinically. This was necessary, because anticoagulating swine with warfarin has proved extremely difficult. ${ }^{14}$ We are reassured, however, by how reproducible this model has been now that we have used it to study various anticoagulation strategies. ${ }^{11,13}$

\section{CONCLUSIONS}

The novel direct thrombin inhibitor dabigatran etexilate was effective for short-term thromboprophylaxis of mechanical heart valves in our porcine model. These animal data provide additional support for clinical trials evaluating dabigatran etexilate as an alternative to warfarin for appropriately selected patients with bileaflet mechanical valve aortic valves.

We thank Joseph A. Rysavy, BS, for his expertise with animal care and DeAnna Haugen for her assistance with thromboelastography. We also thank St Jude Medical for providing the valved conduits for this study.

\section{References}

1. Emery RW, Krogh CC, Arom KV, Emery AM, Benyo-Albrecht K, Joyce LD, et al. The St. Jude Medical cardiac valve prosthesis: a 25-year experience with single valve replacement. Ann Thorac Surg. 2005;79:776-83.

2. Bhatt DL, Fox KA, Hacke W, Berger PB, Black HR, Boden WE, et al. Clopidogrel and aspirin versus aspirin alone for the prevention of atherothrombotic events. N Engl J Med. 2006;354:1706-17.

3. Turpie AG, Eriksson BI, Lassen MR, Bauer KA. Fondaparinux, the first selective factor Xa inhibitor. Curr Opin Hematol. 2003;10:327-32.

4. O'Brien CL, Gage BF. Costs and effectiveness of ximelagatran for stroke prophylaxis in chronic atrial fibrillation. JAMA. 2005;293:699-706.

5. Connolly SJ, Ezekowitz MD, Yusuf S, Eikelboom J, Oldgren J, Parekh A, et al. Dabigatran versus warfarin in patients with atrial fibrillation. $N$ Engl J Med. 2009;361:1139-51. Erratum in: N Engl J Med. 2010;363:1877.

6. Heit JA, Colwell CW, Francis CW, Ginsberg JS, Berkowitz SD, Whipple J, et al. Comparison of the oral direct thrombin inhibitor ximelagatran with enoxaparin as prophylaxis against venous thromboembolism after total knee replacement: a phase 2 dose-finding study. Arch Intern Med. 2001;161:2215-21.

7. Eriksson BI, Borris L, Dahl OE, Haas S, Huisman MV, Kakkar AK, et al. Oral, direct Factor Xa inhibition with BAY 59-7939 for the prevention of venous thromboembolism after total hip replacement. J Thromb Haemost. 2006;4:121-8.

8. Schulman S, Kearon C, Kakkar AK, Mismetti P, Schellong S, Eriksson H, et al. Dabigatran versus warfarin in the treatment of acute venous thromboembolism. N Engl J Med. 2009;361:2342-52.

9. McKellar SH, Thompson JL, Schaff HV. A model of heterotopic aortic valve replacement for studying thromboembolism prophylaxis in mechanical valve prostheses. J Surg Res. 2007;141:1-6.

10. Stangier J, Clemens A. Pharmacology, pharmacokinetics, and pharmacodynamics of dabigatran etexilate, an oral direct thrombin inhibitor. Clin Appl Thromb Hemost. 2009;15(Suppl. 1):9S-16.

11. Thompson JL, Hamner CE, Potter DD, Lewin M, Sundt TM, Schaff HV. Melagatran for thromboprophylaxis after mechanical valve implantation: results in a heterotopic porcine model. J Thorac Cardiovasc Surg. 2007;134:359-65.

12. Hirsh J, Raschke R. Heparin and low-molecular-weight heparin: the Seventh ACCP Conference on Antithrombotic and Thrombolytic Therapy. Chest. 2004; 126:188S-203S

13. McKellar SH, Thompson JL 3rd, Garcia-Rinaldi RF, Macdonald RJ, Sundt TM 3rd, Schaff HV. Short- and long-term efficacy of aspirin and clopidogrel for thromboprophylaxis for mechanical heart valves: an in vivo study in swine. J Thorac Cardiovasc Surg. 2008;136:908-14.

14. Salerno CT, Droel J, Bianco RW. Current state of in vivo preclinical heart valve evaluation. J Heart Valve Dis. 1998;7:158-62.

15. Meurin P, Tabet JY, Weber H, Renaud N, Ben Driss A. Low-molecular-weigh heparin as a bridging anticoagulant early after mechanical heart valve replacement. Circulation. 2006;113:564-9.

16. Bonow RO, Carabello BA, et al., American College of Cardiology/American Heart Association Task Force on Practice Guidelines; Society of Cardiovascular Anesthesiologists; Society for Cardiovascular Angiography and Interventions; 
Society of Thoracic Surgeons. ACC/AHA 2006 guidelines for the management of patients with valvular heart disease: a report of the American College of Cardiology/American Heart Association Task Force on Practice Guidelines (writing committee to revise the 1998 Guidelines for the Management of Patients With Valvular Heart Disease): developed in collaboration with the Society of Cardiovascular Anesthesiologists: endorsed by the Society for Cardiovascular Angiography and Interventions and the Society of Thoracic Surgeons. Circulation. 2006; 114:e84-231. Erratum in: Circulation. 2010;121:e443. Circulation. 2007;115: e409.

17. Jayachandran M, Okano H, Chatrath R, Owen WG, McConnell JP, Miller VM Sex-specific changes in platelet aggregation and secretion with sexual maturity in pigs. J Appl Physiol. 2004;97:1445-52.

18. Thompson JL, Hamner CE, Potter DD, Lewin M, Sundt TM, Schaff HV. Melagatran for thromboprophylaxis after mechanical valve implantation: results in a heterotopic porcine model. J Thorac Cardiovasc Surg. 2007;134(2):359-65.

19. Eriksson BI, Dahl OE, Rosencher N, Kurth AA, van Dijk CN, Frostick SP, et al. Dabigatran etexilate versus enoxaparin for prevention of venous thromboembolism after total hip replacement: a randomised, double-blind, non-inferiority trial. Lancet. 2007;370:949-56. Erratum in: Lancet. 2007;370:2004.

20. Wolowacz SE, Roskell NS, Plumb JM, Caprini JA, Eriksson BI. Efficacy and safety of dabigatran etexilate for the prevention of venous thromboembolism following total hip or knee arthroplasty. A meta-analysis. Thromb Haemost. 2009; 101:77-85.

21. Francis CW. New issues in oral anticoagulants. Hematology Am Soc Hematol Educ Program. 2008;259-65.

\section{Discussion}

Dr Y. Joseph Woo (Philadelphia, $P a$ ). That was very nice talk. Do the valve leaflets move in your model?

Dr McKellar. In this model, they do not. We have verified that under fluoroscopy, and a main reason that we chose this model rather than an intracardiac model is because at 30 days it was much more robust in thrombus formation.

Dr Woo. So have you not created a model system that is exaggerating the thrombus generation? Isn't the relative amount of decrease in thrombus formation in your experimental group always going to look better because your control group is so bad? Most valves in human beings when placed into a native position would not generate that much clot.

Dr McKellar. Correct, and that is why we choose this valve. When we were developing this model, one of our first studies was to compare this with an orthotopic mitral valve position without any anticoagulation, and we found a lot less thrombus in the orthotopic position. We therefore chose the heterotopic position, because the pig grows so rapidly and at 30 days we can keep the pig at minimum growth. So in a short time we can develop a lot of thrombus. I do accept the criticism that this may not completely represent reality of the mechanical valve.

Dr Woo. I just think that it limits the conclusions that you can draw.

Dr McKellar. Thank you for your question.

Dr Michael E. Jessen (Dallas, Texas). I enjoyed your talk, and I really hope that this works out, because I think there is potential in this for patients. One of the difficulties I have with this, though, is that you measured the intensity of anticoagulation in the 2 treatment groups by different methods. One was by anti-factor Xa activity and one was by partial thromboplastin time. So because you were not using exactly the same targets, is it possible that the difference just represents an intensity of anticoagulation, a higher dose of anticoagulation if you like, in one group, and not so much an effect specific to the drug itself?
Dr McKellar. It could, and thank you for your question. This is in large part why we wanted to do a more global evaluation of clot function or clot strength with thromboelastography. According to what is out there in the literature, the anti-Xa levels are fairly well established for the low-molecular weight heparins, whereas for the dabigatran etexilate group it is a little less clear. The best evidence out there for readily available tests for direct thrombin inhibitors is an APPT of 2 to 2.5 times normal, but I understand what you are saying. And we thought that we would see perhaps something like that in the thromboelastographic data, but in fact we saw the reverse. We saw what appears in vitro to be preserved clot function by thromboelastography with dabigatran etexilate versus enoxaparin. As for the anti-Xa level of 0.6 , were we overly anticoagulating in either arm? What we saw on valve thrombus was different from what we saw on thromboelastography, leaving the question open. A good question. Thank you.

Dr Carlos J. Troconis (Santiago, Dominican Republic). There have been several studies in Europe and South Africa about selected cases of On-X valves in aortic replacement without the use of warfarin anticoagulation, treating them only with antiplatelet therapy to avoid thromboembolic events. Do you have any comments about this?

Dr McKellar. We have used this model to look at dual antiplatelet therapy with aspirin and clopidogrel. I know that others around the world have published results of that in human beings. I think that the best place to study that is in patients with good left ventricular function and high flow across a mechanical aortic valve. I know that the On-X people are kind of leading the way in doing some of those trials. But have we done that in human beings? If that is your question, no, we haven't. I do hope that our and other data would push the community toward clinical trials to try this agent in human beings.

Dr Glen Van Arsdell (Toronto, Ontario, Canada). Pigs are notoriously hard to anticoagulate. With unfractionated heparin, you have to give enormous doses. I have 2 questions in that regard. One is the timing of measuring your anti-Xa values. Are you really satisfied that the low-molecular weight heparin was anticoagulating the whole time? The second thing is, if you look at the data on mechanical valves in pregnancy (a hypercoagulable state), lowmolecular weight heparin is not as effective as you would like it to be in terms of preventing valve thrombosis. Does low-molecular weight heparin really anticoagulate well enough in the face of a potentially hypercoagulable physiology?

Dr McKellar. Thank you for your question. According to the American College of Chest Physicians guidelines for deep vein thrombosis and pulmonary embolism prophylaxis or treatment, the best data they have is an anti-Xa of 0.6 at 4 hours after administration, and that is what we duplicated in this study. As to whether there are subtle nuances between the species, granted, I don't have that perfect answer.

Dr. Van Arsdell. So you didn't measure anti-Xa levels right before giving your second dose?

Dr McKellar. Before we did any of the valve implantations, we did a dosing study to find the exact right dose of dabigatran etexilate for our species, as well as the correct dose of enoxaparin, and we did several different doses. The dose we chose consistently yielded a value of 0.6 at 4 hours. 